\title{
INEQUALITIES ON SEVERAL QUASI-ARITHMETIC MEANS
}

\author{
GOU-SHENG YANG ${ }^{1}$, SHUOH-JUNG LIU ${ }^{2, *}$ AND YI-JHE CHEN ${ }^{3}$
}

\begin{abstract}
Inequalities on several quasi-arithmetic means are established by using convexity and concavity.
\end{abstract}

\section{Introduction}

In [3] Y. H. Kim proved the following

Theorem A. Let $a_{i} \geq 0, i=1,2, \ldots, n, x \geq 1$ and $y \geq 0$. Then

$$
\left(\sum_{i=1}^{n} \frac{a_{i}}{n}\right)^{x+y} \leq\left(\sum_{i=1}^{n} \frac{a_{i}^{x}}{n}\right)^{\frac{x+y}{x}} \leq \sum_{i=1}^{n} \frac{a_{i}^{x+y}}{n}
$$

with all equalities holding if and only if all $a_{i}$ are the same.

Let $a_{i}>0, i=1,2, \ldots, n, 0<x \leq 1$, and $y \geq 0$. Then

$$
\left(\prod_{i=1}^{n} a_{i}\right)^{\frac{x+y}{n}} \leq\left(\sum_{i=1}^{n} \frac{a_{i}^{x}}{n}\right)^{\frac{x+y}{x}} \leq\left(\sum_{i=1}^{n} \frac{a_{i}}{n}\right)^{x+y} .
$$

Theorem B. Let $a_{i} \geq 0, i=1,2, \ldots, n, 0<x \leq 1$ and $-x \leq y \leq 0$. Then

$$
\left(\prod_{i=1}^{n} a_{i}\right)^{\frac{x+y}{n}} \leq \sum_{i=1}^{n} \frac{a_{i}^{x+y}}{n} \leq\left(\sum_{i=1}^{n} \frac{a_{i}^{x}}{n}\right)^{\frac{x+y}{x}} \leq\left(\sum_{i=1}^{n} \frac{a_{i}}{n}\right)^{x+y},
$$

with all equalities holding if and only if all $a_{i}$ are the same.

Theorem C.(corrected) Let $a_{i}>0, i=1,2, \ldots, n, x \geq 1$, and $-x \geq y \geq-2 x$. Then

$$
\left(\sum_{i=1}^{n} \frac{a_{i}^{x}}{n}\right)^{\frac{x+y}{x}} \leq\left(\sum_{i=1}^{n} \frac{a_{i}}{n}\right)^{x+y} \leq\left(\prod_{i=1}^{n} a_{i}\right)^{\frac{x+y}{n}} \leq \sum_{i=1}^{n} \frac{a_{i}^{x+y}}{n} .
$$

Corresponding author: Shuoh-Jung Liu.

2010 Mathematics Subject Classification. 26D07; 26D15.

Key words and phrases. Convex functions, concave functions, means, strictly increasing functions, strictly decreasing functions. 
Theorem D.(corrected) Let $a_{i}>0, i=1,2, \ldots, n, x \geq 1$, and $-2 x \geq y$. Then

$$
\left(\sum_{i=1}^{n} \frac{a_{i}^{x}}{n}\right)^{\frac{x+y}{x}} \leq\left(\sum_{i=1}^{n} \frac{a_{i}}{n}\right)^{x+y} \leq\left(\prod_{i=1}^{n} a_{i}\right)^{\frac{x+y}{n}} \leq\left(\sum_{i=1}^{n} \frac{1}{n a_{i}^{x}}\right)^{\frac{-(x+y)}{x}} \leq \sum_{i=1}^{n} \frac{a_{i}^{x+y}}{n} .
$$

In [1] S. Abramovich, J. Pečarić and S. Varošance have proved several generalizations of Kim's results, one of which is the following

Theorem E. Let $x \geq 1, y \geq 0$, and $\frac{x+y}{x} \geq x$. If $a_{i} \geq 0, i=1,2, \ldots, n$, then

$$
\left(\sum_{i=1}^{n} \frac{a_{i}}{n}\right)^{x+y} \leq\left(\sum_{i=1}^{n} \frac{a_{i}^{x}}{n}\right)^{\frac{x+y}{x}} \leq\left(\sum_{i=1}^{n} \frac{a_{i}^{\frac{x+y}{x}}}{n}\right)^{x} \leq \sum_{i=1}^{n} \frac{a_{i}^{x+y}}{n} .
$$

Inequalities (6) follow also from Theorem C and Corollary 4 in [1].

In this paper we give some generalizations of the above inequalities by using convexity and concavity.

\section{Main results}

Throughout,let $f, g:[a, b] \rightarrow[c, d], a_{i} \in[c, d], 0 \leq \alpha_{i} \leq 1, i=1,2, \ldots, n$ and $\sum_{i=1}^{n} \alpha_{i}=1$.

\section{Lemma 1. If one of the following conditions holds}

(i) $g$ is strictly increasing, $g^{-1} \circ f$ is convex and strictly monotone on $[a, b]$;

(ii) $g$ is strictly decreasing, $g^{-1} \circ f$ is concave and strictly monotone on $[a, b]$ then

$$
f\left(\sum_{i=1}^{n} \alpha_{i} f^{-1}\left(a_{i}\right)\right) \leq g\left(\sum_{i=1}^{n} \alpha_{i} g^{-1}\left(a_{i}\right)\right) .
$$

If one of the following conditions holds

(iii) $g$ is strictly increasing, $g^{-1} \circ f$ is concave and strictly monotone on $[a, b]$;

(iv) $g$ is strictly decreasing, $g^{-1} \circ f$ is convex and strictly monotone on $[a, b]$

then

$$
g\left(\sum_{i=1}^{n} \alpha_{i} g^{-1}\left(a_{i}\right)\right) \leq f\left(\sum_{i=1}^{n} \alpha_{i} f^{-1}\left(a_{i}\right)\right)
$$

Proof. We will prove the result for case (i), where $g^{-1} \circ f$ is strictly increasing on $[a, b]$, a similar argument establishes the results for other cases. If $x, y \in[a, b]$ and $x<y$,since $g^{-1} \circ f$ is strictly increasing on $[a, b]$, we have $g^{-1}(f(x))=\left(g^{-1} \circ f\right)(x)<\left(g^{-1} \circ f\right)(y)=g^{-1}(f(y))$. 
Since $g$ is strictly increasing,it follows that $f(x)<f(y)$, so that $f$ is strictly increasing on $[a, b]$ and $f^{-1}$ exists.

Since $g^{-1} \circ f$ is convex on $[a, b]$, we have

$$
\left(g^{-1} \circ f\right)\left(\sum_{i=1}^{n} \alpha_{i} f^{-1}\left(a_{i}\right)\right) \leq \sum_{i=1}^{n} \alpha_{i}\left(g^{-1} \circ f\right)\left(f^{-1}\left(a_{i}\right)\right)=\sum_{i=1}^{n} \alpha_{i} g^{-1}\left(\left(f \circ f^{-1}\right)\left(a_{i}\right)\right)=\sum_{i=1}^{n} \alpha_{i} g^{-1}\left(a_{i}\right)
$$

Since $g$ is strictly increasing, it follows from the above result that

$$
f\left(\sum_{i=1}^{n} \alpha_{i} f^{-1}\left(a_{i}\right)\right) \leq g\left(\sum_{i=1}^{n} \alpha_{i} g^{-1}\left(a_{i}\right)\right)
$$

This completes the proof.

Proposition 1. Suppose $a_{i}>0, i=1,2, \ldots, n$. Then

(i) If $x+y \geq 0, x \geq 1$ or $x<0, x+y \leq 0$ or $0<x \leq 1, x+y \leq 0$, then

$$
\left(\sum_{i=1}^{n} \alpha_{i} a_{i}\right)^{x+y} \leq\left(\sum_{i=1}^{n} \alpha_{i} a_{i}^{x}\right)^{\frac{x+y}{x}}
$$

(ii) If $x+y \geq 0,0<x \leq 1$ or $x<0, x+y \geq 0$ or $x \geq 1, x+y \leq 0$, then

$$
\left(\sum_{i=1}^{n} \alpha_{i} a_{i}^{x}\right)^{\frac{x+y}{x}} \leq\left(\sum_{i=1}^{n} \alpha_{i} a_{i}\right)^{x+y}
$$

Proof. Let $f(t)=t^{x+y}$ and $g(t)=t^{\frac{x+y}{x}}$. Then (9) follows from (7) and (9') follows from (8).

Proposition 2. Suppose $a_{i}>0, i=1,2, \ldots, n$. Then

(i) If $x y \geq 0, x \neq 0$ or $x>0, y \leq 0, x+y \leq 0$ or $x<0, y \geq 0, x+y \geq 0$, then

$$
\left(\sum_{i=1}^{n} \alpha_{i} a_{i}^{x}\right)^{\frac{x+y}{x}} \leq \sum_{i=1}^{n} \alpha_{i} a_{i}^{x+y}
$$

(ii) If $x>0, y \leq 0, x+y \geq 0$ or $x<0, y \geq 0, x+y \leq 0$, then

$$
\left(\sum_{i=1}^{n} \alpha_{i} a_{i}^{x}\right)^{\frac{x+y}{x}} \geq \sum_{i=1}^{n} \alpha_{i} a_{i}^{x+y} .
$$

Proof. Let $f(t)=t^{\frac{x+y}{x}}$ and $g(t)=t$. Then (10) follows from (7) and (10')follows from (8).

Remark 1. Suppose $a_{i}>0, i=1,2, \ldots, n$, it follows from (9) and (10) that, if $x \geq 1, y \geq 0$ or $x<0, y \leq 0$, or $0<x \leq 1, x+y \leq 0$, then

$$
\left(\sum_{i=1}^{n} \alpha_{i} a_{i}\right)^{x+y} \leq\left(\sum_{i=1}^{n} \alpha_{i} a_{i}^{x}\right)^{\frac{x+y}{x}} \leq \sum_{i=1}^{n} \alpha_{i} a_{i}^{x+y},
$$


which extends the inequality (1) in Theorem A with $\alpha_{i}=\frac{1}{n}, i=1,2, \ldots, n$.

Proposition 3. Suppose $a_{i}>0, i=1,2, \ldots, n$. Then

$$
\prod_{i=1}^{n} a_{i}^{\alpha_{i} x} \leq \sum_{i=1}^{n} \alpha_{i} a_{i}^{x}, \forall x
$$

Proof. Let $f(t)=t^{\frac{1}{x}}$ and $g(t)=e^{t}$. Then (11) follows from (8).

We note that the inequality (11) reduces to

$$
\text { geometric mean } \leq \text { arithmetric mean, }
$$

if we choose $x=1$ and reduces to

$$
\text { harmonic mean } \leq \text { geometric mean, }
$$

if we choose $x=-1$.

Remark 2. Suppose $a_{i}>0, i=1,2, \ldots, n$, it follows from $\left(9^{\prime}\right),\left(10^{\prime}\right)$ and (11) that, if $0<x \leq 1, y \leq$ $0, x+y \geq 0$, then

$$
\prod_{i=1}^{n} a_{i}^{\alpha_{i}(x+y)} \leq \sum_{i=1}^{n} \alpha_{i} a_{i}^{x+y} \leq\left(\sum_{i=1}^{n} \alpha_{i} a_{i}^{x}\right)^{\frac{x+y}{x}} \leq\left(\sum_{i=1}^{n} \alpha_{i} a_{i}\right)^{x+y},
$$

which is the inequality (3) in Theorem B with $\alpha_{i}=\frac{1}{n}, i=1,2, \ldots, n$.

Proposition 4. Suppose $a_{i}>0, i=1,2, \ldots, n$. Then

(i) If $\frac{x+y}{x} \leq 0$, then

$$
\prod_{i=1}^{n} a_{i}^{\alpha_{i}(x+y)} \geq\left(\sum_{i=1}^{n} \alpha_{i} a_{i}^{x}\right)^{\frac{x+y}{x}}
$$

(ii) If $\frac{x+y}{x} \geq 0$, then

$$
\prod_{i=1}^{n} a_{i}^{\alpha_{i}(x+y)} \leq\left(\sum_{i=1}^{n} \alpha_{i} a_{i}^{x}\right)^{\frac{x+y}{x}}
$$

Proof. Let $f(t)=t^{\frac{x+y}{x}}$ and $g(t)=e^{t}$. Then (12) follows from (7) and (12') follows from (8).

Remark 3. Suppose $a_{i}>0, i=1,2, \ldots, n$, it follows from $\left(9^{\prime}\right)$ and $\left(12^{\prime}\right)$ that, if $0<x \leq 1, x+y \geq 0$, then

$$
\prod_{i=1}^{n} a_{i}^{\alpha_{i}(x+y)} \leq\left(\sum_{i=1}^{n} \alpha_{i} a_{i}^{x}\right)^{\frac{x+y}{x}} \leq\left(\sum_{i=1}^{n} \alpha_{i} a_{i}\right)^{x+y}
$$


which extends the inequality (2) in Theorem A with $\alpha_{i}=\frac{1}{n}, i=1,2, \ldots, n$.

Proposition 5. Suppose $a_{i}>0, i=1,2, \ldots, n$. Then

(i) If $x \leq 0$, then

$$
\prod_{i=1}^{n} a_{i}^{\alpha_{i} x} \geq\left(\sum_{i=1}^{n} \alpha_{i} a_{i}\right)^{x}
$$

(ii) If $x \geq 0$, then

$$
\prod_{i=1}^{n} a_{i}^{\alpha_{i} x} \leq\left(\sum_{i=1}^{n} \alpha_{i} a_{i}\right)^{x}
$$

Proof. Let $f(t)=t^{x}$ and $g(t)=e^{t}$. Then (13) follows from (7) and (13') follows from (8).

Remark 4. Suppose $a_{i}>0, i=1,2, \ldots, n$, it follows from (9'),(11) and (13) that, if $x \geq 1, x+y \leq 0$, then

$$
\left(\sum_{i=1}^{n} \alpha_{i} a_{i}^{x}\right)^{\frac{x+y}{x}} \leq\left(\sum_{i=1}^{n} \alpha_{i} a_{i}\right)^{x+y} \leq \prod_{i=1}^{n} a_{i}^{\alpha_{i}(x+y)} \leq \sum_{i=1}^{n} \alpha_{i} a_{i}^{x+y}
$$

which extends the inequality (4) in Theorem C with $\alpha_{i}=\frac{1}{n}, i=1,2, \ldots, n$.

Remark 5. Suppose $a_{i}>0, i=1,2, \ldots, n$, it follows from (12) and (13') that, if $x<0, x+y \geq 0$, then

$$
\left(\sum_{i=1}^{n} \alpha_{i} a_{i}^{x}\right)^{\frac{x+y}{x}} \leq \prod_{i=1}^{n} a_{i}^{\alpha_{i}(x+y)} \leq\left(\sum_{i=1}^{n} \alpha_{i} a_{i}\right)^{x+y}
$$

Proposition 6. Suppose $a_{i}>0, i=1,2, \ldots, n$. Then

(i) If $\frac{x+y}{x} \geq 0$, then

$$
\left(\sum_{i=1}^{n} \alpha_{i} a_{i}^{-x}\right)^{\frac{-(x+y)}{x}} \leq \prod_{i=1}^{n} a_{i}^{\alpha_{i}(x+y)}
$$

(ii) If $\frac{x+y}{x} \leq 0$, then

$$
\left(\sum_{i=1}^{n} \alpha_{i} a_{i}^{-x}\right)^{\frac{-(x+y)}{x}} \geq \prod_{i=1}^{n} a_{i}^{\alpha_{i}(x+y)}
$$

Proof. Let $f(t)=t^{\frac{-(x+y)}{x}}$ and $g(t)=e^{t}$. Then (14) follows from (7) and (14') follows from (8). 
Remark 6. Suppose $a_{i}>0, i=1,2, \ldots, n$, it follows from (9),(13) and (14) that, if $x \geq 1, x+y \geq 0$, then

$$
\left(\sum_{i=1}^{n} \alpha_{i} a_{i}^{-x}\right)^{\frac{-(x+y)}{x}} \leq \prod_{i=1}^{n} a_{i}^{\alpha_{i}(x+y)} \leq\left(\sum_{i=1}^{n} \alpha_{i} a_{i}\right)^{x+y} \leq\left(\sum_{i=1}^{n} \alpha_{i} a_{i}^{x}\right)^{\frac{x+y}{x}} .
$$

Proposition 7. Suppose $a_{i}>0, i=1,2, \ldots, n$. Then $\frac{x+y}{x} \leq-1$ or $\frac{x+y}{x} \geq 0$, then

$$
\sum_{i=1}^{n} \alpha_{i} a_{i}^{x+y} \geq\left(\sum_{i=1}^{n} \alpha_{i} a_{i}^{-x}\right)^{\frac{-(x+y)}{x}}
$$

Proof. Let $f(t)=t^{\frac{-(x+y)}{x}}$ and $g(t)=t$. Then (15) follows from (7).

Remark 7. Suppose $a_{i}>0, i=1,2, \ldots, n$, it follows from $\left(9^{\prime}\right),\left(13^{\prime}\right),\left(14^{\prime}\right)$ and (15) that, if $x \geq$ $1, \frac{x+y}{x} \leq-1$, then

$$
\left(\sum_{i=1}^{n} \alpha_{i} a_{i}^{x}\right)^{\frac{x+y}{x}} \leq\left(\sum_{i=1}^{n} \alpha_{i} a_{i}\right)^{x+y} \leq \prod_{i=1}^{n} a_{i}^{\alpha_{i}(x+y)} \leq\left(\sum_{i=1}^{n} \alpha_{i} a_{i}^{-x}\right)^{\frac{-(x+y)}{x}} \leq \sum_{i=1}^{n} \alpha_{i} a_{i}^{x+y}
$$

which is the inequality (5) in Theorem D with $\alpha_{i}=\frac{1}{n}, i=1,2, \ldots, n$.

Proposition 8. Suppose $a_{i}>0, i=1,2, \ldots, n$. Then

(i) If $x \geq 1$ or $x<0$, then

$$
\left(\sum_{i=1}^{n} \alpha_{i} a_{i}^{\frac{x+y}{x}}\right)^{x} \leq \sum_{i=1}^{n} \alpha_{i} a_{i}^{x+y}
$$

(ii) If $0<x \leq 1$, then

$$
\left(\sum_{i=1}^{n} \alpha_{i} a_{i}^{\frac{x+y}{x}}\right)^{x} \geq \sum_{i=1}^{n} \alpha_{i} a_{i}^{x+y}
$$

Proof. Let $f(t)=t^{x}$ and $g(t)=t$. Then (16) follows from (7) and (16') follows from (8).

Proposition 9. Suppose $a_{i}>0, i=1,2, \ldots, n$. Then

(i) If $x>0, x+y \geq x^{2}$ or $x>0, x+y \leq 0$ or $x<0 \leq x+y \leq x^{2}$, then

$$
\left(\sum_{i=1}^{n} \alpha_{i} a_{i}^{x}\right)^{\frac{x+y}{x}} \leq\left(\sum_{i=1}^{n} \alpha_{i} a_{i}^{\frac{x+y}{x}}\right)^{x}
$$

(ii) If $x>0,0 \leq x+y \leq x^{2}$ or $x<0, x+y \geq x^{2}$ or $x<0, x+y \leq 0$, then

$$
\left(\sum_{i=1}^{n} \alpha_{i} a_{i}^{x}\right)^{\frac{x+y}{x}} \geq\left(\sum_{i=1}^{n} \alpha_{i} a_{i}^{\frac{x+y}{x}}\right)^{x} .
$$


Proof. Let $f(t)=t^{\frac{x+y}{x}}$ and $g(t)=t^{x}$. Then (17) follows from (7) and (17') follows from (8).

Remark 8. Suppose $a_{i} \geq 0, i=1,2, \ldots, n$, it follows from (9), (16) and (17), that if $x \geq 1, x+y \geq$ $x^{2}$, then

$$
\left(\sum_{i=1}^{n} \alpha_{i} a_{i}\right)^{x+y} \leq\left(\sum_{i=1}^{n} \alpha_{i} a_{i}^{x}\right)^{\frac{x+y}{x}} \leq\left(\sum_{i=1}^{n} \alpha_{i} a_{i}^{\frac{x+y}{x}}\right)^{x} \leq \sum_{i=1}^{n} \alpha_{i} a_{i}^{x+y}
$$

These last inequalities follow also from Corollary 4 in [1] where general results are stated. The special case $\alpha_{i}=\frac{1}{n}, i=1,2, \ldots, n$ is stated there as Corollary 6 .

Remark 9. Suppose $a_{i}>0, i=1,2, \ldots, n$, it follows from (9'), $\left(16^{\prime}\right)$ and (17'), that if $0<x \leq$ $1,0 \leq x+y \leq x^{2}$, then

$$
\left(\sum_{i=1}^{n} \alpha_{i} a_{i}\right)^{x+y} \geq\left(\sum_{i=1}^{n} \alpha_{i} a_{i}^{x}\right)^{\frac{x+y}{x}} \geq\left(\sum_{i=1}^{n} \alpha_{i} a_{i}^{\frac{x+y}{x}}\right)^{x} \geq \sum_{i=1}^{n} \alpha_{i} a_{i}^{x+y}
$$

Proposition 10. If we choosing $f(t)=t^{\frac{1}{x}}, g(t)=t^{\frac{1}{y}}, t>0, x \neq 0, y \neq 0$, then it follows from (7) that if $0<x \leq y$ or $x<0<y$ or $x \leq y<0, a_{i}>0, i=1,2, \ldots, n$, then

$$
\left(\sum_{i=1}^{n} \alpha_{i} a_{i}^{x}\right)^{\frac{1}{x}} \leq\left(\sum_{i=1}^{n} \alpha_{i} a_{i}^{y}\right)^{\frac{1}{y}}
$$

which shows that the mean of order $t$, defined by $M_{t}(a, \alpha)=\left(\sum_{i=1}^{n} \alpha_{i} a_{i}^{t}\right)^{\frac{1}{t}}$ is a nondecreasing function of $t$ for $-\infty<t<\infty$ (see [2, p17] or [4, p15]).

\section{Acknowledgements}

The present investigation was supported, in part, by the National Science Council of the Republic of China under Grant NSC 99-2811-M-033-014.

\section{References}

[1] S. Abramovich, J. Pečarić and S. Varošance, Comparison Theorems between several quasi- arithmetic means, Math. Ineq. Appl., 7 (2004), 1-6.

[2] E. F. Beckenbach, R. Bellman, Inequalities, Spring-Verlag, Berlin-Newyork, 1961.

[3] Y. H. Kim, Refinements and Extensions of an Inequality, J. Math. Anal. Appl., 245(2000), 628-632.

[4] D. S. Mitrinović, J. E. Pečarić and A. M. Fink, Classical and New Inequalities in Analysis, Kluwer Academic, Dordrecht/Boston/London, 1993.

${ }^{1}$ Department of Mathematics Tamkang University, Tamsui 25137, Taiwan, Republic of China.

E-mail:005490@math.tku.edu.tw 
${ }^{2}$ Department of Applied Mathematics, Chung Yuan Christian University, Chung-Li 32023, Taiwan, Republic of China.

E-mail: liusolong@gmail.com

${ }^{3}$ Department of Mathematics Tamkang University, Tamsui 25137, Taiwan, Republic of China.

E-mail: phills132002@yahoo.com.tw 